\title{
Diabetes in pregnancy
}

\author{
Robert Fraser
}

\section{Introduction}

Perinatal mortality in association with maternal diabetes in pregnancy, which were as high as $50 \%$ at the time of the Second World War, have fallen dramatically. By the early 1980 s in most units offering some specialised supervision, perinatal mortality was no higher than for non-diabetic women in the same hospital.

The clinical problems which remain include: maintaining the low level of perinatal mortality in the face of changing obstetric practice and fashion; continuing attempts to reduce the mortality and morbidity associated with the excess of congenital malformations in the offspring of women with diabetes; and the even more intractable problem of preventing the birth of hyperinsulinaemic, macrosomic fetuses with the multiple problems they face as newborns.

I will comment briefly on the vexed area of screening for, and diagnosis of, gestational diabetes mellitus. The other areas of interest to obstetricians and diabetic physicians caring for these women - the effect of pregnancy on the progression of the disease and its complications, and the association between gestational diabetes and subsequent development of adult diabetes - will not be considered.

\section{Diabetes diagnosed before conception (pre-gestational diabetes)}

In the United Kingdom population insulin dependent diabetes (IDDM), often of childhood origin, predominates among cases of diabetes predating pregnancy. In populations where higher mean parity and corresponding tendency to reproduction at a later age are more common, non-insulin dependent diabetes (NIDDM) may predominate in numerical terms. High rates of NIDDM complicating pregnancy are typically seen in emerging economies such as the Middle East and in immigrant populations in the United Kingdom, particularly those from the Indian subcontinent.

In the United Kingdom pre-gestational diabetes complicates two to 10 pregnancies in a thousand. Gestational diabetes, or carbohydrate intolerance diagnosed for the first time during pregnancy, may complicate 10 to 50 pregnancies in every thousand. This latter figure is only tentative and depends on variable ascertainment rates.

\section{PERINATAL MORTALITY}

Without specialist care the perinatal mortality associated with maternal diabetes can be extremely high. There are many contributing factors. Major congenital malformations are four to six times more common in women who are diabetic at the time of conception than in non-diabetic women. Many of these malformations are lethal either in utero or in the newborn period. Diabetic pregnancy is associated with complications such as polyhydramnios and an excess of pre-eclampsia which may lead to spontaneous or iatrogenic pre-term birth. ${ }^{1}$ The offspring of diabetic mothers have an excess of hyaline membrane disease at all stages of gestation, and newborn respiratory problems have in the past been a major factor in the excess perinatal mortality rates.

Between four and 10 babies in every 1000 may suddenly die in utero, particularly in the later weeks of pregnancy. These deaths have often been in macrosomic fetuses. Why these babies die is still not known, although acute on chronic hypoxia is emerging as the most likely possible cause. To reduce the high numbers of death in utero elective early delivery became established practice. This was advocated at 38, or more commonly, 37 weeks' gestation either by induction of labour or by elective caesarean section, although in some centres induction as early as 35 weeks was recommended. The result of this practice, perhaps predictably, was a number of deaths associated with pulmonary immaturity. These deaths may have exceeded the number of lives saved by the policy. The pendulum seems to be swinging back towards a policy of allowing pregnancies to continue until the onset of spontaneous labour, but whether this policy is any safer will be the subject of later comment.

Other major contributing factors to perinatal mortality (and morbidity) were birth trauma, particularly asphyxiation, or fetal injury, when shoulder dystocia obstructed delivery, and other complications of the newborn period such as severe hypoglycaemia.

Although it had been suggested for many years that improved diabetic control might reduce rates of perinatal mortality, it was the 1972 keynote publication of the retrospective study of diabetic pregnancy by Karlsson and Kjellmer which was instrumental in changing practice. ${ }^{2}$ These authors performed a 10 year analysis of 179 Swedish women in whom a systematic attempt had been made to document the quality of diabetic control in the 
Table 1 Congenital malformations in infants of diabetic mothers ${ }^{\star}$

\begin{tabular}{lc}
\hline & Ratio of incidencest \\
\hline Spina bifida & 2 \\
Anencephaly & 3 \\
Cardiac anomalies & 4 \\
Low gut atresias & 3 \\
Renal anomalies & 5 \\
Situs inversus & 84 \\
Caudal regression & 252
\end{tabular}

${ }^{\star}$ Modified form Mills $\mathrm{et} \mathrm{al}^{3}$

†Ratio of incidences: frequency of malformation in fetuses of diabetic women compared with that of similar malformations in non-diabetics.

weeks before delivery. Their results showed that there was a linear relation between quality of diabetic control and absolute levels of perinatal mortality, with the most poorly controlled women (mean blood glucose of $>7 \cdot 7$ $\mathrm{mol} / \mathrm{l}$ ) experiencing perinatal mortality of $24 \%$, rates that were almost as bad as those reported 20 years earlier. Those women with mean glucose concentrations below $5.5 \mathrm{mmol} / 1$ had a $3.8 \%$ rate of perinatal mortality which was no higher than non-diabetic women studied over the same time period. The effects of these results were profound. Indeed, if blood glucose is controlled in the third trimester, and average daily glucose concentrations are below 6 mmol, very low perinatal mortality is seen. The main benefits of the intervention are probably in terms of reduced polyhydramnios, pre-eclampsia, and pre-term labour. Later delivery is associated with less serious respiratory distress.

The newborn of the diabetic mother has also certainly benefited from the dramatic improvements which have been seen in neonatal care over the past 15 years, particularly in terms of respiratory support and prevention of hypoglycaemia.

\section{CONGENITAL MALFORMATIONS}

In collected series major structural malformations occur four to six times more often in the fetuses of diabetic women than in those of non-diabetics. The pattern of malformations comprises an excess of all the common types of structural malformations, including neural tube defects, gut atresias, and perhaps particularly, serious cardiac malformations. The search for a typical pattern of anomalies associated with maternal diabetes has been inconclusive apart from two rather rare syndromes which are numerically much more common in diabetics - caudal regression and situs inversus (table 1) (figure). Despite the relatively high ratios of incidences for caudal regression, this is an extremely rare malformation, occurring in only two in every 1000 diabetics. Although many hypotheses have been generated to explain this finding, once again the major determinant of congenital malformation in the fetus of a diabetic mother seems to be related to the quality of the mother's blood glucose control, not in late pregnancy but around the time of conception and embryogenesis. A rather clever observation was reported by Miller and colleagues in the early $1980 \mathrm{~s},{ }^{4}$ when it became possible to measure glycosylated haemoglobin in the laboratory. It occurred to them that glycosylated haemoglobin measured in the first weeks of pregnancy in diabetic women would reflect the quality of periconceptional glucose control. Their study of 112 women showed that almost all the babies subsequently found to have congenital malformations were in the upper half of the distribution of glycosylated haemoglobin measurements. This information was converted into a prospective trial by Fuhrmann, ${ }^{5}$ among others. His uncontrolled studies performed in the former East Germany suggested that women subjected to a rigorous programme of tight periconceptional diabetic control had a rate of congenital malformation no higher than that of non-diabetic women. Those who did not participate in the regimen had congenital malformation rates of $7.5 \%$, close to the expected figure. Other groups have explored this approach with somewhat less convincing results. At present no formal randomised trial of good control $v$ 'intensive' control of diabetes in the periconceptional period has been undertaken. Clearly there are disadvantages to the intensive regimen, not least that the woman who is planning a pregnancy would not know for how long she would have to continue it. While she was in intensive control she would be at risk of the serious problem of insulin induced hypoglycaemia. Of interest, in this regard, are the detailed studies of Mills and colleagues. ${ }^{6}$ They showed that although in women controlling their diabetes scrupulously in the periconceptional period the congenital malformation rate was $4.9 \%$ compared with $9 \%$ for diabetic women not involved in the programme, the malformation rate in the well

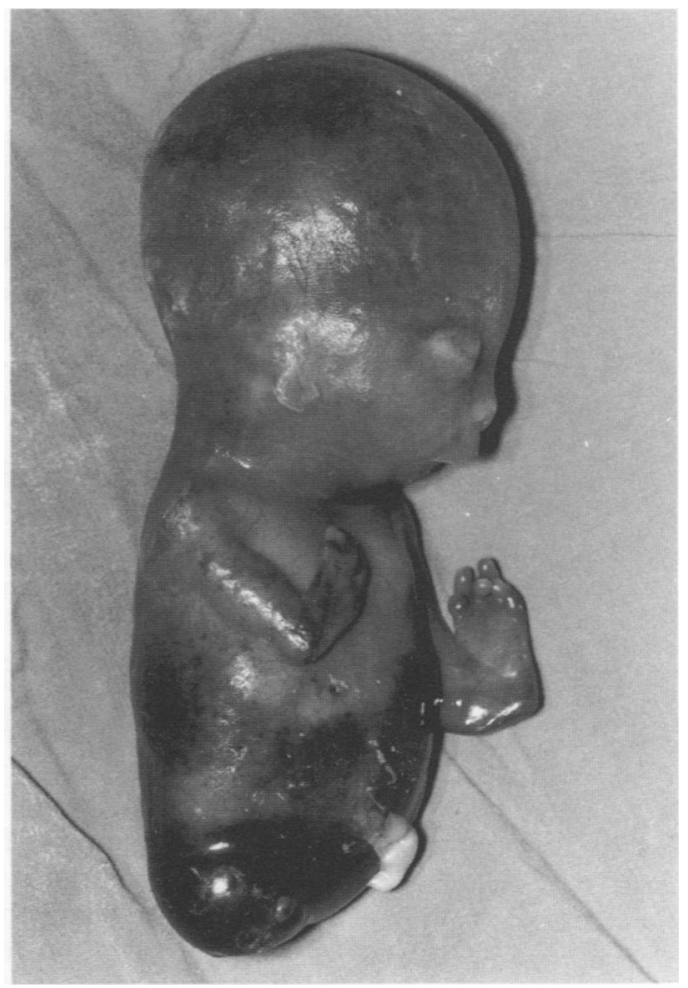

Multiple congenital malformations, including severe caudal regression, in fetus of a woman with IDDM, identified by ultrasound scan at 19 weeks. Pregnancy was terminated. 
Table 2 Diabetic control and birth weight centiles

\begin{tabular}{lll}
\hline $\begin{array}{l}\text { Mean pre-prandial } \\
\text { blood glucose (mmoll) } \\
\text { (weeks 30-38 of gestation) }\end{array}$ & \multicolumn{2}{l}{ Birth weight centile for gestational age } \\
\cline { 2 - 3 } \cline { 2 - 2 } & 10th-90th & $>90$ th \\
\hline$<4 \cdot 0$ & 3 & 1 \\
$<5 \cdot 0$ & 1 & 3 \\
$<6 \cdot 0$ & 3 & 1 \\
$>6 \cdot 0$ & 2 & 0 \\
Total & 9 & $5(35 \%)$ \\
\hline
\end{tabular}

Fourteen consecutive cases of IDDM had regular blood sugar profiles from 30-38 weeks of gestation (R Fraser, unpublished observations.) Half of the women who achieved very tight control $(<5.0 \mathrm{mmol} / \mathrm{l})$ also had large for gestational age infants.

controlled diabetics was still twice that of nondiabetic control women. Furthermore, the quality of control as reflected by glycosylated haemoglobin results in early pregnancy did not show the clear correlation with malformations reported in the earlier study by Miller. ${ }^{4}$ Although studies in small animals have suggested a correlation between hypoglycaemia and malformation, this is not reported in human beings, apart from a few anecdotal cases. In one recent series only one of 46 women who had severe hypoglycaemia had a malformed baby. ${ }^{7}$

On a practical note it should be borne in mind that with advances in the antenatal diagnosis of structural and chromosomal malformations and the widespread acceptance of termination of pregnancy, it is becoming quite rare for diabetic women in the United Kingdom to give birth to babies with major structural malformations.

\section{THE MACROSOMIC FETUS}

The classic study of Karlsson and Kjellmer confirmed the association between perinatal mortality and poor third trimester diabetic control, but perhaps, surprisingly, failed to make a similar connection between maternal control in late pregnancy and birth weight. ${ }^{2}$ The birth weight distribution was such that some of the largest babies were born to some of the best controlled mothers, and vice versa.

There is a paradox here, however, which might contribute to this statistical observation, in that longstanding complicated diabetes with macrovascular pathology in the mother is associated with intrauterine growth retardation. It is generally felt that poor placental perfusion results from inadequate expansion of the blood supply to the placental bed because of arteriosclerosis. The effect would mean, perhaps, that some growth retarded babies would be born to the worst controlled diabetic mothers, but it would not explain why some of the largest babies were born to some of the best controlled mothers. Our own observations in this area are summarised by the results of a series of diabetics shown in table 2 in whom attempts were made to provide optimal diabetic control, aiming for a mean blood glucose of less than $6 \mathrm{mmol} / \mathrm{l}$ during the day. In each of these cases regular profiles were obtained from 30 weeks' gestation to about 38 weeks, when delivery was undertaken electively. A third of the babies had a birth weight above the 90th centile for gestational age; four of the five large babies were born to mothers among the eight best controlled. Our experience in this small series is typical of the experience of others in larger series, and it is rare to find reported macrosomia rates below $30 \%$.

The finding of these high rates of macrosomia, despite apparently good diabetic control, has been the source of much speculation. For all practical purposes, although glucose crosses the placenta freely by facilitated diffusion, insulin does not, and the insulin secretory kinetics of the mother and fetus, each on their own side of the placenta, are independent of each other. In the case of the insulin dependent diabetic where the insulin is exogenous the usual administrative difficulties obtain. The essential problem is that exogenous subcutaneous insulin or even intravenous insulin cannot be administered in a pattern which mimics the in vivo situation. This means that despite what would appear to be almost perfect diabetic control, judged by intermittent diurnal profiling, very high concentrations of plasma glucose may be experienced transiently after meals. Within the overall pattern of concentration dependent glucose flux across the placenta, there is evidence of a degree of idiosyncracy in fetal uptake. These two factors may mean that the hyperinsulinaemia in the fetus is a simple response to excess glucose transfer across the placenta. Many workers are unwilling to accept this simple cause and effect hypothesis and have searched for other mechanisms.

Insulin is a primitive hormone which influences all nutrient subclasses. Insulin deficiency not only leads to hyperglycaemia but also to high concentrations of amino acids, free fatty acids, and a secondary rise in ketone bodies, all of which may become important alternate fetal substrates and could therefore contribute to macrosomia. A good deal of effort has been put into evaluating the possible effects of these alternate substrates on the assumption that they could be operating to cause macrosomia despite optimal blood glucose control, but there is both theoretical and experimental evidence to suggest that the simple explanation is the correct one. The theoretical evidence has already been alluded to in that it is very difficult to know in an individual case what the true diurnal pattern of glycaemic excursion is and, more particularly, what the rate of glucose transfer of the individual placental system might be in different people with the same level of glycaemia. The experimental evidence that glucose control may be at fault in cases of macrosomia arises from the work of Roversi's group in Italy. They developed a system of thrice daily insulin which was increased until clinical hypoglycaemia was obtained at each time point. ${ }^{8}$ The dose which had not provoked hypoglycaemia was referred to as the 'maximum tolerated dose' and this was administered with relatively fixed calorie intakes for the remainder of pregnancy. The levels of maternal glycaemia were to all intents and purposes ignored, but where they were measured they were found to be about $25 \%$ lower on diurnal profiles than 
the target levels referred to earlier. The policy of maximum tolerated doses of insulin produced a fairly normal birth weight distribution, with only $8 \%$ of babies born above the 90 th centile of weight for gestational age. The problem, therefore, for those who cannot accept that simple glucose transfer is the explanation for hyperinsulinaemia or macrosomia is that they must invalidate rather than simply ignore Roversi's results.

Among those fetuses destined to be born macrosomic, hyperinsulinaemia is an almost constant feature. It can be detected antenatally by measuring liquor insulin concentrations. Not only can fetal hyperinsulinaemia be confirmed, but there is evidence that once recognised target orientated insulin treatment can reverse it before birth. ${ }^{9}$ So far these observations have not been translated into general clinical practice. Antenatal diagnosis of fetal hyperinsulinaemia can also be made by cordocentesis, although the relative risks of this procedure compared with simple amniocentesis do not seem to be justified. There is some evidence that cordocentesis can be used to identify chronic hypoxaemia in the hyperinsulinaemic fetus, and this might assist decision making about elective delivery to prevent intrauterine death. Tissue hypoxia in the fetus may also be reflected in erythropoetin concentrations. ${ }^{10}$ Erythropoetin concentrations in liquor or cord blood may turn out to be a marker of chronic fetal hypoxaemia. The lack of a widely available erythropoetin assay limits clinical use at present.

For those reluctant to undertake invasive testing, or without access to an insulin assay, the sensitivity of a single measure of fetal abdominal circumference for predicting macrosomia is $56 \%$, with a specificity of $96 \% .{ }^{11}$ Again, the possibility of target orientated insulin treatment could be entertained with an antenatal diagnosis of macrosomia based on ultrasound scans. A failure of growth curves, between head circumference and abdominal circumference, to continue to divaricate might be evidence of therapeutic success. This is because the macrosomia typical of hyperinsulinaemia affects different organs selectively. The large fetus of a diabetic mother is different from the constitutionally large fetus of the same weight in that the liver, muscles, and subcutaneous fat organs are all considerably increased in the diabetic fetus whereas brain growth is not excessive. In the constitutionally large fetus, while head circumference and abdominal circumference are relatively large, their ratio is not distorted. It is of clinical importance that because brain growth is not excessive, whereas somatic growth does exceed normal centiles, the mechanism exists for shoulder dystocia. The head can pass through the birth canal unobstructed, but the shoulders become arrested at the pelvic inlet. Imaging techniques, both ultrasound scans and computed tomography, have now been applied in an attempt to predict shoulder dystocia, and therefore to avoid the potentially fatal complication caused by performing a caesarean section. Shoulder widths above
$14 \mathrm{~cm}$ are associated with birth weights above $4200 \mathrm{~g}$ and might be judged to be an indication for caesarean section. ${ }^{12}$ The other obvious associations with macrosomia, which might be detected by ultrasound examination, are increased placental volume and increased liquor volume. The ultrasound scan detection of polyhydramnios depends on the finding of at least one liquor pocket above $8 \mathrm{~cm}$ in depth.

There is a debate in obstetric circles about the timing of delivery in pregnancies complicated by maternal diabetes. With falling perinatal mortality, some authorities have suggested that induction at 37 or 38 weeks is no longer necessary and indeed is not in the mother's interest because failed induction is associated with a high risk of caesarean section. It is true that spontaneous labour is less likely to result in caesarean section, but delaying delivery in these cases is probably not justified because of our continuing inability to identify the fetus at risk of intrauterine death. It seems unforgivable to preside over the avoidable death of an apparently normal fetus, after subjecting diabetic women to the rigours of an antenatal care that includes intensified glucose control and frequent hospital visits.

During labour or in preparation for caesarean section there is an argument for maintaining good diabetic control - usually with balanced glucose and insulin infusions. The fetus of the diabetic mother has an adult type insulin secretory kinetic pattern and may respond to maternal hyperglycaemia in labour with fetal hyperinsulinaemia, leading to hypoglycaemia in the first four hours of life. ${ }^{13}$

The other current argument involves paediatricians in decisions as to whether routine admissions to the special care baby unit are necessary. Some modern series suggest that neonatal complication rates are now so low that routine admission would be unjustified. Admission policy should therefore be based on condition at birth and subsequent development of biochemical hypoglycaemia or respiratory distress. Such a policy has obvious advantages in terms of bonding and perhaps would contribute to successful continuation rates of breast feeding. A recently published series from Sweden of national data on IDDM complicating pregnancy, in 491 cases studied over a five year period, reports rates of idiopathic respiratory distress syndrome of $1.6 \%$, transient tachypnoea of the newborn of $3.3 \%$, symptomatic hypoglycaemia of $8 \%$, hyperbilirubinaemia of $16.3 \%$, polycythemia of $2.2 \%$, and an Apgar score of less than seven at five minutes in $3 \cdot 4 \% .{ }^{14}$ This complication rate seems particularly low and indeed the expectation of complications in our experience is closer to that of Berk et al (table 3). ${ }^{15}$ Close scrutiny of this table is of interest, not least because of the high rate of neonatal complications in babies who are judged to be appropriate for gestational age. 'Normal birth weight' has often been the justification for allowing such babies to go to the routine postnatal ward on the first day of life. 
Table 3 Neonatal morbidity in appropriate for gestational age $(A G A)$ and large for gestational age ( $L G A)$ neonates ${ }^{\star}$

\begin{tabular}{lccl}
\hline & $L G A$ & $A G A$ & p Value \\
\hline Polycythaemia & $24 \%$ & $7 \%$ & $<0 \cdot 01$ \\
Hyperbilirubinaemia & $30 \%$ & $13 \%$ & $<0 \cdot 05$ \\
Hypoglycaemia & $60 \%$ & $50 \%$ & NS \\
RDS & $10 \%$ & $12 \%$ & NS \\
Birth asphyxia & $16 \%$ & $35 \%$ & NS
\end{tabular}

NS: differences not significant

Modified from Berk et al ${ }^{15}$

\section{Gestational diabetes}

The uncertainty which still surrounds this subject is surprising after the extraordinary amount of scientific and clinical effort spent on it by obstetricians over the past 30 years. The term used to refer to carbohydrate intolerance, diagnosed during pregnancy, which resolved after pregnancy. This definition has been updated by the 3rd International Workshop Conference on gestational diabetes mellitus to 'Carbohydrate metabolism of variable severity with onset or first recognition during pregnancy'. ${ }^{16}$ The new definition of course includes IDDM, and NIDDM diagnosed for the first time during pregnancy, as well as the reversible state referred to earlier. The problem was a practical one - that reversibility could be confirmed only by postnatal glucose tolerance testing, and a considerable proportion of women defaulted from such testing.

In brief, there are problems with ascertainment of gestational diabetes mellitus, comparability of different diagnostic tests for it, and indeed interpretation criteria. Most Europeans currently favour the $75 \mathrm{~g}$ oral glucose tolerance test proposed by the WHO Expert Committee, with diagnosis based on a two hour value. Americans generally favour the American Diabetic Association criteria based on a $100 \mathrm{~g}$ load and a three hour test in which any two values have to exceed a normal range for the diagnosis to be made. The WHO criteria have been criticised because they fail to take account of physiological changes in glucose concentrations, both at fasting and at two hours which are seen in normal pregnancy, and may overestimate the true incidence of gestational diabetes mellitus. Despite this, they only diagnose about half the cases of gestational diabetes mellitus (or as the WHO styled it, impaired glucose tolerance) compared with the American criteria. ${ }^{17}$ Some authorities have reviewed the situation and concluded that the condition does not exist as a pathological state justifying screening, ${ }^{18}$ but others have come to the conclusion that the American requirement for two abnormal values is too rigorous because macrosomia and perinatal morbidity can be detected in women with only one abnormal value. ${ }^{19}$

In summary, it is likely that no two hospitals will have an exactly identical programme of screening for, diagnosis of, or treatment of gestational diabetes mellitus.

PERINATAL MORTALITY AND MORBIDITY Although the classic studies of O'Sullivan reported an excess perinatal mortality in women with gestational diabetes, more particularly in those who were overweight and over 25 years of age, ${ }^{20}$ more recent series have reported no excess perinatal mortality in gestational diabetes mellitus. This may relate to increased awareness and supervision, or indeed treatment.

There does not seem to be the same excess of congenital malformation and this would make sense if fetopathy was related to hyperglycaemia in early pregnancy, a condition which is usually absent in gestational diabetes mellitus.

The problem of excess fetal growth or fetal hyperinsulinaemia does seem to be present in women diagnosed as having gestational diabetes mellitus, although of course the prevalence of this complication depends to some extent on the diagnostic criteria. The matter is complicated further by the fact that women with potential diabetes tend to have an excess of heavy birth weight infants, despite normal glucose tolerance testing. ${ }^{21}$ In collected series there is an approximate doubling of birth weight over the 90th centile in women with untreated gestational diabetes mellitus, and this excess can be reduced by diet, treatment, and insulin treatment. The effect of insulin in reducing birth weight does not seem to be directly related to mean plasma glucose concentrations.

The real concern here is that because of inadequate screening a proportion of hyperinsulinaemic fetuses may be unrecognised until they develop complications either during delivery or in the early newborn period. Our own work in this area is consistent with that of others and suggests that among unselected babies born over the 90th centile of weight for gestational age, about a quarter are hyperinsulinaemic and therefore at risk. ${ }^{22}$ We have noted, however, that hyperinsulinaemia also occurs rarely in appropriate for gestational age newborns with no predisposing maternal history or glucose tolerance abnormality.

\section{Summary of obstetric and medical} management of pregnant diabetic women PRE-PREGNANCY COUNSELIING

It goes without saying that any known diabetic should have the benefit of pre-pregnancy counselling, including contraception or sterilisation, as appropriate, or investigation and treatment of infertility. There is a concern that for adolescent girls this type of counselling is not as readily available as it should be. It may be that they fall between two stools in the transfer between paediatric diabetic clinics and adult diabetic clinics, each thinking that sex education and pre-pregnancy counselling is the province of the other.

At pre-pregnancy counselling we advise contraception to be continued, with intensification of insulin treatment to achieve glycosylated haemoglobin concentrations in the nondiabetic range. This type of supervision is often best undertaken in diabetes centres under the supervision of diabetes specialist nurses. Women with NIDDM planning a pregnancy 
who are maintained on oral hypoglycaemics should be carefully reviewed and the institution of insulin treatment before pregnancy considered. Fundoscopy should be performed by a suitably experienced practitioner and any pre-proliferative or proliferative retinopathy be treated with photocoagulation, preferably before discontinuing contraception. Established nephropathy should be well documented both in terms of average amounts of proteinuria and creatinine clearance. With severe grades of nephropathy (creatinine clearance of $<40 \mathrm{ml} /$ minute) pre-pregnancy counselling might raise the question of whether childbearing should be deferred until after successful transplantation, if that option seems imminent.

\section{CARE IN EARLY PREGNANCY}

It is important to try and confirm pregnancy as soon as possible in diabetic women; sensitive pregnancy tests can be followed up by early abdominal or vaginal ultrasound scanning. In diabetics a condition known as 'early growth delay' may operate and prejudice subsequent measurements of maturity which are important for antenatal diagnostic screening and for timing of elective delivery. The possibility that early growth delay is an artefact induced by delayed ovulation does not seem to have been satisfactorily excluded. ${ }^{23}$

Early scanning will confirm fetal viability and gestational age. Diabetic women should be offered all available antenatal diagnostic screening for neural tube defects and chromosomal abnormalities, with recourse to amniocentesis if these tests show abnormal results. More important is to offer a detailed structural scan at 19 to 20 weeks; the ultrasonographer should be informed that the woman is diabetic and make sure that experience in cardiac scanning and limb assessment is available.

Antenatal care is almost always managed on an outpatient basis, although most expert centres prefer the visits to be to hospital clinics rather than general practitioner or community midwives' surgeries. Obstetric assessment includes surveillance for development of preeclampsia, polyhydramnios, and abnormalities of fetal growth, the latter measured by serial ultrasound scans. Diabetic care consists of insulin adjustments which aim to maintain mean pre-prandial blood glucose (usually assessed on sticks with meters at home) below $6 \mathrm{mmol} / \mathrm{l}$ if this can be achieved without clinical hypoglycaemia.

Admission to hospital may be indicated if outpatient control fails to achieve satisfactory profiles.

The management of pre-term labour presents problems because $\beta$ sympathometics are almost certainly contraindicated due to the acute loss of diabetic control which has sometimes been associated with their use. There is a similar although probably quantitatively lesser risk associated with the mother taking steroids when pre-term delivery seems likely. On balance it seems reasonable to administer steroids when indicated but it should ideally be done with the knowledge of the consultant responsible for diabetic care so that appropriate adjustment in insulin dose can be made.

Biochemical tests for lung maturity based on the lecithin:sphingomyelin ratio are notoriously unreliable in diabetic women below 37 weeks' gestation. In current practice the indications for this type of testing even in diabetics have become few indeed, perhaps because of improvements in ultrasound dating of pregnancy.

\section{TIMING OF DELIVERY}

For the reasons reviewed we still favour elective delivery at around 38 weeks' gestation although practice varies and in some units 40 weeks is becoming the norm. For induction of labour cervical favourability can be induced by local prostaglandin treatment and indeed this can shorten the length of the latent phase of labour, particularly in the patient pregnant for the first time. This seems worthwhile as prolonged labour in a woman prone to ketosis may be dangerous to both mother and fetus. Modern insulin and glucose regimens for management of labour correspond to standard regimens for intraoperative and postoperative management of the diabetic. Either dextrose infusions with fixed insulin and potassium contents or a piggy-back arrangement using a dextrose drip and a separate insulin infusion pump (adjustable) can be used. Glucose concentrations in labour are generally held to be between 5 and $8 \mathrm{mmol} / \mathrm{l}$.

There is a low threshold to opt for caesarean section for the reasons described earlier and this may be particularly the case where there has been a previous caesarean section, or where there is a breech presentation, or an ultrasound diagnosis of macrosomia. When vaginal delivery is anticipated this should be supervised by an experienced midwife and it is a sensible precaution to have a registrar or other experienced obstetrician in the room to assist should there be unexpected difficulties with the shoulders. In most units the on-call paediatric team will be happy to attend, both in case resuscitation should be needed and to examine the newborn to provide reassurance about normality for the new parents. Policy about indications for transfer to the special care baby unit for observation or intravenous dextrose should preferably be agreed with the parents in the antenatal period.

\section{MANAGEMENT AFTER DELIVERY}

Diabetic women having caesarean section will normally be given prophylaxis with antibiotics and routine subcutaneous heparin and antiembolism stockings. They should be managed with intravenous dextrose insulin regimens until they return to a normal diet. The insulin regimen can almost immediately be restarted at the pre-pregnant dose. Non-insulin dependent diabetics treated with insulin and gestational diabetics will generally not require insulin after the delivery and can be managed with serial measurements of the blood glucose 
by sticks and a meter. If the newborn baby of a diabetic mother is returned to the postnatal ward a regimen for supervision of both feeding and glucose monitoring should be clearly understood and enforced by paediatricians and midwives or nurses in charge of the ward.

\section{Conclusions}

There are sufficient problems with the obstetric management of the pregnant diabetic patient to justify continuing clinical research. It is possible, however, to identify modifications of practice which may improve outcome.

1. Improved pre-pregnancy counselling and dissemination of information about the effects of diabetes to women at risk.

2. Attention to diabetic control, particularly with the involvement of diabetes centres and diabetes nurse specialists, may reduce the incidence of congenital malformations and fetal hyperinsulinaemia and macrosomia.

3. Improved antenatal screening and the use of selective termination, where acceptable, can 'reduce' perinatal mortality and morbidity.

4. Improved diagnostic testing using ultrasound scanning and invasive techniques may become available to identify the hyperinsulinaemic fetus and also secondary hypoxaemia. Coupled with steroid treatment in reducing the risk of respiratory distress syndrome, it may be possible to rationalise policies for timing of delivery. Such a future scenario would have a significant impact on short term neonatal morbidity and may very well improve long term outcomes of the newborn of diabetic mothers.

1 Greene MF, Hare JW, Krache M, Phillippe M, Barss VA Saltzman DH, et al. Prematurity among insulin-requiring diabetic gravid women. Am f Obstet Gynecol 1989; 161 106-11.

2 Karlsson K, Kjellmer I. The outcome of diabetic pregnancies in relation to the mother's blood sugar level. $A m$ Obstet Gynecol 1972; 112: 213-20.

3 Mills JL, Baker L, Goldman AS. Malformation in infants of diabetic mothers occurs before the seventh gestational week. Diabetes 1979; 28: 292-3.

4 Miller E, Hare JW, Cloherty JP, Dunn PJ, Gleeson RE, Soeldner JS, et al. Elevated maternal $\mathrm{HbA}_{1 \mathrm{c}}$ in early preg nancy and major congenital anomalies in infants of diabetic mothers. N Engl F Med 1981; 304: 1331-4.
5 Fuhrmann K, Reiher H, Semmler K, Fischer F, Fischer M, Glockner E. Prevention of congenital malformations in infants of insulin dependent diabetic mothers. Diabetic Care 1983; 6: 219-23.

6 Mills JL, Knopp RH, Simpson JL, Iovanovic-Peterson L Metzger BE, Holmes LB, et al. Lack of relation of increased malformation rates in infants of diabetic mothers to glycemic control during organogenesis. N Engl f Med 1988; 318: 671-6.

7 Steel JM, Johnstone FD, Hepburn DA, Smith AF. Can prepregnancy care of diabetic women reduce the risk of abnormal babies? BMF 1990; 301: 1070-4.

8 Roversi GD, Gargiulo M, Nicolini U, Pedretti E, Marini A, Barbarani V, et al. A new approach to the treatment of diabetic pregnant women. Am f Obstet Gynecol 1979; 135 567-76

9 Weiss PAM. Prophylactic insulin in gestational diabetes. Obstet Gynecol 1988; 71: 951.

10 Salvesen DR, Brudenell JM, Snijders RJM, Ireland RM, Nicolaides KH. Fetal plasma erythropoetin in pregnancies complicated by maternal diabetes mellitus. Am $\mathcal{f}$ Obstet Gynecol 1993; 168: 88-94.

11 Bochner CJ, Medearis AC, Williams J, Castro L, Hobel CJ Wade ME. Early third trimester ultrasound screening in gestational diabetes to determine the risk of macrosomia and labour dystocia at term. Am $\mathcal{F}$ Obstet Gynecol 1987 157: 703-8.

12 Kitzmiller JL, Mall JC, Gin GD, Hendricks SK, Newman RB, Scheerer L. Measurement of fetal shoulder width with computed tomography in diabetic women. Obste Gynecol 1987; 70: 941-5.

13 Miodovnik M, Mimouni F, Tsang RC, Skillman C, Siddigi TA, Butler JB, et al. Management of the insulindependent diabetic during labor and delivery: Influences on neonal outcome Am 7 Perinatol 1987; 106-14.

14 Hanson U, Persson B. Outcome of pregnancies complicated by type I insulin-dependent diabetes in Sweden: Acute pregnancy complications, neonatal mor

15 Berk MA, Mimouni F, Miodovnik M, Hertzberg V, Valuck $\mathrm{J}$. Macrosomia in infants of insulin dependent mothers Pediatrics 1989; 83: 1029-34.

16 American Diabetes Association. Summary and recommendations of the 3rd International Workshop-Conference on Gestational Diabetes Mellitus. Diabetes 1991; 40 (supp 2): $1-201$.

$17 \mathrm{Li} \mathrm{DH}$, Wong VCW, O'Hoy $\mathrm{KM}$, Ma HK. Evaluation of the WHO criteria for the $75 \mathrm{~g}$ oral glucose tolerance test in pregnancy. Br $\mathcal{f}$ Obstet Gynaecol 1987; 94: test in $847-50$.

18 Jarrett RJ. Gestational diabetes: a non-entity? BMf 1993 ; 306: $37-8$.

19 Langer O, Brusman L, Anyaegbunam A, Mazze R. The significance of one abnormal glucose tolerance test value on adverse outcome of pregnancy. Am $\mathcal{F}$ Obstet Gynecol 1987 157: 758-63.

20 O'Sullivan JB, Charles D, Mahon CM, Dandrow RV. Gestational diabetes and perinatal mortality rate. $A m \mathscr{f}$ Obstet Gynecol 1973; 116: 901-4.

21 Ford FA, Bruce C, Fraser RB. Fetal macrosomia in potential diabetics with normal oral glucose tolerance: a
case-control study. Br 7 Obstet Gynaecol 1990; 97: 957-62.

22 Stanley KP, Fraser RB, Milner M, Bruce C. Cord insulin and $\mathrm{C}$-peptide - Distribution in an unselected population. Br f Obstet Gynaecol 1992; 99: 512-5.

23 Steel JM, Corrie JET, Johnstone FD. Assessment of gestation by salivary progesterone and its relevance to the concept of early growth delay in diabetic pregnancy. Diabetologia 1984; 27: 334A. 